\title{
Predictors of hyperglycaemic individuals who do not follow up with physicians after screening in Japan: A cohort study.
}

\section{$\operatorname{AUTHOR}(\mathrm{S}):$}

Tsujimura, Yuka; Takahashi, Yoshimitsu; Ishizaki, Tatsuro; Kuriyama, Akira; Miyazaki, Kikuko; Satoh, Toshihiko; Ikeda, Shunya; Kimura, Shinya; Nakayama, Takeo

\section{CITATION:}

Tsujimura, Yuka ... [et al]. Predictors of hyperglycaemic individuals who do not follow up with physicians after screening in Japan: A cohort study.. Diabetes research and clinical practice 2014, 105(2): 176-184

\section{ISSUE DATE:}

2014-08

URL:

http://hdl.handle.net/2433/189892

\section{RIGHT:}

(c) 2014 Elsevier Ireland Ltd.; This is not the published version. Please cite only the published version.; この論文は出版社版でありません。引 用の際には出版社版をご確認ご利用ください。 


\section{TITLE PAGE}

\section{Title:}

Predictors of Hyperglycaemic Individuals Who do not Follow up with Physicians after Screening in Japan: a Cohort Study

\section{Author names:}

Yuka Tsujimura, MD, $\mathrm{MPH}^{1}$

Yoshimitsu Takahashi, $\mathrm{PhD}^{1}$

Tatsuro Ishizaki, MD, $\mathrm{PhD}^{2}$

Akira Kuriyama, MD, MPH ${ }^{1}$

Kikuko Miyazaki, $\mathrm{PhD}^{1}$

Toshihiko Satoh, MD, MPH, $\mathrm{PhD}^{3}$

Shunya Ikeda, MD, $\mathrm{PhD}^{4}$

Shinya Kimura ${ }^{5}$

Takeo Nakayama, MD, $\mathrm{PhD}^{1}$

\section{Affiliations:}

1. Department of Health Informatics, Kyoto University School of Public Health,

Yoshidakonoe-cho, Sakyo-ku, Kyoto City, 606-8501, Japan 
2. Tokyo Metropolitan Institute of Gerontology, 35-2 Sakaecho, Itabashi-ku, Tokyo, 173-0015, Japan

3. School of Social Informatics, Aoyama Gakuin University, 5-10-1 Fuchinobe, Chuo-ku, Sagamihara City, 252-5258, Japan

4. Department of Pharmaceutical Sciences, School of Pharmacy, International University and Health Welfare, 2600-1 Kitakanemaru, Ohtawara City, 324-8501, Japan

5. Japan Medical Data Center Co., Ltd., 3-1, Koujimachi, Chiyoda-ku, Tokyo, 102-0083, Japan

\section{Author contributions:}

Yuka Tsujimura: design, data management, analysis and interpretation of data, drafting article

Yoshimitsu Takahashi: design, interpretation of data, drafting article

Tatsuro Ishizaki: design, interpretation of data, drafting article

Akira Kuriyama: design, interpretation of data, drafting article

Kikuko Miyazaki: design, drafting article

Toshihiko Satoh: design, interpretation of data, drafting article

Shunya Ikeda: design, interpretation of data, drafting article

Shinya Kimura: design, data collection, drafting article

Takeo Nakayama: design, analysis and interpretation of data, drafting article

\section{Corresponding Author:}


Yuka Tsujimura, MD, MPH, Department of Health Informatics, Kyoto University School of

Public Health, Yoshidakonoe-cho, Sakyo-ku, Kyoto City, 606-8501, Japan

Telephone/FAX: 81-75-753-9477/ 81-75-753-9478

E-mail: tsujimura.yuka.67z@st.kyoto-u.ac.jp / yukanakatani11@gmail.com

\section{Word Count:}

Text; 3914 words, 4 Tables, 2 Figures

\section{Grant support:}

This work was partly supported by a grant-in aid from the Ministry of Education, Culture, Sports,

Science and Technology, Japan [Challenging Exploratory Research 24659320]. 


\section{ABSTRACT}

Aims: Although people screened as being hyperglycaemic often fail to follow up with physicians for clinical assessment, epidemiologic findings on the frequency and predictors of not following up (hereafter, “no follow-up”) are lacking. The purpose of this study was to examine the no follow-up rate with physicians after screening for diabetes and its predictors.

Methods: We assessed cases of no follow-ups with physicians within six months after screening based on medical claims data from employee-based social health insurance programs in Japan, for people aged 20 to 68 years from 2005 to 2010.

Results: Among 3,878 screened participants with hyperglycemia, 2,527 (65\%) did not follow up with their physicians within six month after screening. Multiple logistic regression analysis revealed that younger age and lower blood glucose level predicted no follow-ups among both men and women, while lower body mass index and negative proteinuria also predicted no follow-ups among men. Treatment for dyslipidaemia facilitated follow-ups among both genders, and treatment for hypertension or depression facilitated follow-ups among men.

Conclusions: Approximately two thirds of individuals screened as having hyperglycemia did not follow up with their physicians within six months after screening. Predictors of no follow-ups 
were younger age and milder hyperglycemia. Treatment of comorbidities tended to facilitate

follow-ups.

\section{Keywords}

diabetes; screening; no follow-up; Japan 


\section{Introduction}

Diabetes mellitus is associated not only with the occurrence of vascular complications, but also with impaired quality of life and increased health care costs ${ }^{1-3}$. In 2013, 382 million people had diabetes in the world; this number was expected to rise to 592 million by $2035^{4}$. The total number of excess deaths attributable to diabetes in 2010 was estimated to be 3.96 million in the age group of 20-79 years ${ }^{5}$. Approximately $17 \%$ of the Japanese population is estimated to have diabetes, and this number increases every year 6 .

The effectiveness of screening for diabetes remains controversial ${ }^{7-14}$. In Japan, a unique system of general health screening that includes glucose testing was established by the Health and Medical Service Act for the Aged in 1983. Screenings have been conducted primarily at worksites or local community facilities, and patients who screen positively are advised to follow up with a physician. Thus, following up with a physician is a requisite for this screening system to work. A Japanese national survey based on self-report revealed that 39\% of people with diabetes had not been treated $^{6}$, indicating that many individuals who are potentially diabetic neither visited nor followed up with physicians.

A previous cross-sectional study based on self-report suggested that younger people and those with lower risk were more likely to drop out from screenings ${ }^{15}$. Another cross-sectional study reported that individuals with type 2 diabetes who suffer from depression poorly adhere to 
self-care ${ }^{16}$. Yet, no study has reported longitudinal findings using objective data to address this issue. To this end, we conducted a cohort study to examine the characteristics of people who did not follow up with physicians for clinical assessment after they were screened as being hyperglycaemic, with a focus on age, severity of hyperglycemia and comorbidities.

\section{Materials and Methods}

\section{Setting}

In Japan, all people have been insured under universal health coverage since 1961, which primarily consists of employee-based and community-based social health insurance programs ${ }^{17}$. The Industrial Safety and Health Law enacted in 1972 requires that public screening services be provided at worksites or local community facilities, rather than taking place at a family or attending physician's office as in the United States and other countries.

In order to obtain a confirmed diagnosis or to initiate treatment after screening, screened individuals with a positive test result must follow up with physicians (Figure 1). In Japan, standard screening for adults was implemented by the Law of Health and Medical Services for the Elderly from 1983 to 2008, although it is now under the control of the Act on Assurance of Medical Care for Elderly People, which was enacted in 2008. Our data include both periods. Both laws instruct those whose examined values exceed cut-off values to visit physicians, and most health insurance programs advise them to do so simply by mail. Disease management, including 
education, monitoring or feedback, for screened people is not proactively carried out by the government Rather, disease management differs by the particular health insurance system. Screened people generally receive instructions to consult with a physician within about two months of the screening, although a medical institution or timing of the visit is not specified. That is, in Japan, although health insurance plans provide screening to insured people, the plans fall short of designating a hospital. This reflects the disconnect between health insurance programs and hospitals, although regular health check-ups are the norm and mass screening is provided for everyone at school and work or in the community ${ }^{18}$.

In 2007, the Ministry of Health, Labour and Welfare proposed a standard program for screening and health guidance ${ }^{19}$, and screenings are currently conducted according to this program. Screenings are held by each health insurance program, although the frequency differs by program. Most screenings are held during the day, and blood and/or urine samples are collected early in the morning. Screened participants are instructed to fast from 9 p.m. the night before to the end of screening, although the degree of compliance with these instructions is unclear. In general, there are two approaches to the screening. Either people visit a specifically-designated facility to receive the check-up, or screeners visit the worksite. For worksite-based screenings in our study, vehicles carrying medical testing equipment visited each worksite and carried out the screenings. Depending on the insurance program, some participants visited a facility designated by the program to undergo screening. For community-based screenings, non-employed people typically 
visit local facilities.

Screening services follow several general steps that represent the analytical framework for diabetes screening ${ }^{8}$. We reworked this framework to adapt it for diabetes screening in Japan

(Figure 1). As mentioned above, Japanese screenings are held mainly at community facilities or worksites (Step 1 in Figure 1), followed by visiting physicians (Step 2 in Figure 1). For the screening program to work effectively, people need to adhere to all steps.

\section{Study design and data source}

We carried out a retrospective cohort study using administrative data from both health insurance claims and screenings from Japan Medical Data Center Co., Ltd. (JMDC, Tokyo, Japan). JMDC obtained health insurance claims and screening data from several employee-based social health insurance plans and constructed the JMDC medical database (JMDC-MDB). This database includes 530,000 insured people and their dependents, mainly company employees and their family members, from January 2004 to December 2010. Health insurance claims were anonymously linked with screening data in JMDC-MDB ${ }^{20}$. The database provided patient demographics, screening results, test orders, treatments, prescribed drugs and hospital diagnoses. The locality of the health insurance plans or the identities of the insured were not available to the researchers. Under these circumstances, all employees and family members were automatically eligible for the screening and were encouraged to attend it. 
The Institutional Review Board of the Kyoto University Graduate School and Faculty of

Medicine Ethics Committee approved the study protocols (E1017).

\section{Study participants}

Study participants were screened individuals with untreated hyperglycemia. The study cohort included 220,409 employees and their dependent family members, from 2005 to 2010. Of the 220,409 participants, we excluded 118,765 who did not attend a screening (screening attendance rate: $46 \%$ ) and 9,749 who did not undergo glycated haemoglobin $\left(\mathrm{HbA}_{1 \mathrm{c}}\right)$ or fasting glucose tests. Of the remaining 91,895 participants, 5,834 participants (6.3\%) were considered hyperglycaemic $\left(\mathrm{HbA}_{1 \mathrm{c}} \geq 6.5 \%[48 \mathrm{mmol} / \mathrm{mol}]\right.$, or fasting glucose $\left.\geq 126 \mathrm{mg} / \mathrm{dl}\right)$. Of these, 1,927 were being treated with antihyperglycaemic agents. When participants were identified as hyperglycaemic twice or more, we defined "hyperglycaemic at screening" as the last time of screening in the study period. We excluded people who were born before $1940(n=19)$ because of missing birth dates, and people who were under 20 years at the time of screening $(n=10)$. Data from the remaining 3,878 participants (3,420 men and 458 women) were analysed (Figure 2).

\section{Outcome measurement}

"Follow-up” refers to the initial visit to a physician within six months after the screening, accompanied by an examination of $\mathrm{HbA}_{1 \mathrm{c}}$ and/or glucose. When a participant who screened 
positive did not follow up with a physician, this was designated as "no follow-up”, which was regarded as the primary outcome measurement. We determined the indicated visit as being for treatment of diabetes or not according to whether further glycaemia testing, an examination of $\mathrm{HbA}_{1 \mathrm{c}}$ and/or glucose was done at the visit. Those who visited a physician for the other diseases (e.g., common cold) were excluded. Whether patients were on track for treatment or not is beyond the scope of the present study, and thus "follow-up" also included those who made even a single visit to a physician. For the most part, screenings are performed once (although in some cases, twice a year). Accordingly, the primary outcome measure for this study was "no follow-up with a physician within six month after screening.” For sensitivity analysis, we also examined no follow-up with a physician within three and 12 months of screening.

\section{Baseline variables}

Demographic characteristics and screening data for participants were obtained directly from JMDC-MDB. Body mass index (BMI $\left.\left[\mathrm{kg} / \mathrm{m}^{2}\right]\right)$ was calculated from weight and height measured during screening as a measure for obesity. $\mathrm{HbA}_{1 \mathrm{c}}$ values determined by the Japan Diabetes Society (JDS) method was $0.4 \%$ lower than NGSP values ${ }^{21}$, so we converted them to NGSP values.

We identified comorbidities using information from health insurance claims on hypertension treatment, dyslipidaemia, proteinuria and depression. Hypertension and dyslipidaemia are chronic 
metabolic diseases that, like hyperglycemia, require drug therapy and lifestyle modification. We hypothesized that participants being treated for these diseases may be more likely to follow up with physicians if they were hyperglycaemic at screening. Proteinuria was chosen as a marker of possible chronic kidney disease. A previous study reported that depression is associated with non-adherence to diabetes self-care among patients with type 2 diabetes ${ }^{16}$, implying that depression precludes engaging in desirable health behaviours. Therefore, treatment for depression was included as a potential predictor of non-adherence to follow-ups. We defined "hypertension” as prescription of hypertension medication within three months prior to screening: C02, 03, 04, 07, 08, or 09 in the Anatomical Therapeutic Chemical Classification System²2. Similarly, we defined “dyslipidaemia” as prescription of medication for dyslipidaemia (C10), and “depression” as prescription of medication for depression (N06A or N06BA). Participants who were diagnosed with hypertension, dyslipidaemia and/or depression were excluded if their comorbidities were left untreated. “Glycosuria” was classified as either negative (urine dipstick, negative) or positive (urine dipstick, trace or above). Proteinuria was also classified as negative (urine dipstick, negative or trace) or positive (urine dipstick, 1+ or above). For patients who already had hypertension or dyslipidaemia, we examined whether they visited the same clinics for hyperglycemia as for these other diseases. Men and women were analysed separately. Smoking (yes/no) and frequency of alcohol consumption (everyday/sometimes/rarely) were self-reported and missing in a considerable proportion of the participants (23\% and 36\%, 
respectively); participants without these data were excluded from the analysis. Given the lack of data regarding education level, marital status, race or ethnicity, type of employee-based social health insurance plan and insured/dependent individual were used as surrogates of these socio-economic variables.

\section{Statistical analysis}

We identified mean or median values, and frequencies of explanatory variables that were potential predictors or confounders, stratified by gender. We excluded variables if there were more than $20 \%$ missing data. For the remaining variables, we used mean values for missing data of continuous variables, and "no" for dichotomous variables.

We calculated the frequency of no follow-ups after screening, and used logistic regression models to estimate odds ratios (ORs) and 95\% confidence intervals (CIs) to determine predictors. As explanatory variables, we used baseline variables for which there were less than $20 \%$ missing data (age, gender, $\mathrm{HbA}_{1 \mathrm{c}}$, fasting glucose, $\mathrm{BMI}$, glycosuria, proteinuria, haemoglobin, hypertension, dyslipidaemia, depression, type of health insurance plans and insured/dependent status at the health insurance plans). Multiple logistic regression analyses were performed following analyses using age-adjusted models. We also analysed data for two additional follow-up periods: within three months and within 12 months. The statistical models were examined using the area under the receiver operating characteristic (ROC) curve (C statistic). 
Tests of statistical significance were two-tailed, with an $\alpha$-level of 0.05 . All analyses were performed with STATA, version 11.1 (Stata Corp., College Station, TX, USA).

\section{Results}

\section{Screening attendance rate and baseline characteristics of participants with hyperglycemia}

Among 101,644 of 220,409 cohort members who participated in a screening (attendance rate:

46\%), 91,895 underwent glucose testing, of whom 5,834 (6.3\%) were hyperglycaemic and 3,907

(4.3\%) were hyperglycaemic without treatment. There were 3,878 study participants remaining after excluding those under 20 years of age or born in 1940 or before (Figure 2). HbA1c and fasting glucose levels were used to identify participants with suspected diabetes (i.e., if they had values higher than the cut-off). Among the 3,878 study participants, HbA1c and fasting glucose were examined in 3,784 (97.6\%) and 3,363 (86.7\%) participants, respectively. For 94 (2.4\%) participants, only data for fasting glucose were available.

We used age, gender, $\mathrm{HbA}_{1 \mathrm{c}}$, fasting glucose, $\mathrm{BMI}$, glycosuria, proteinuria, haemoglobin, hypertension, dyslipidaemia, depression, type of health insurance plan, and insured/dependent status in the health insurance plan as variables; these data were complete or missing less than $20 \%$

\section{(Table 1).}

Table 1 shows the baseline characteristics of 3,878 participants screened as hyperglycaemic. More 
men than women had higher haemoglobin levels (15.2 g/dl vs. $13.1 \mathrm{~g} / \mathrm{dl})$, were more likely to smoke (40.4\% vs. 7.6\%), consumed alcohol more frequently (14.4\% vs. 2.0\% for everyday drinking), had depression more often (2.3\% vs. 6.6\% for depression), had hypertension more often (16.9\% vs. 13.8\%) and were insured (99.97\% vs. 49.1\%).

\section{Frequency of no follow-up}

Of the 3,878 hyperglycaemic participants, 2,527 (65.2\%) did not follow up with physicians within six months (Figure 2, Table 1). Men were less likely to follow up with physicians than women during the three, six, and 12-month follow-up periods (74.9\% vs. $67.0 \%, 65.9 \%$ vs. $59.4 \%$ and 58.2\% vs. 53.7\% for no follow-ups, respectively). When stratified by HbA1c levels, $>40 \%$ of participants with an HbA1c of $8.0 \%$ (64 mmol/mol) or over, and 35\% of participants with HbA1c of $10.0 \%$ (86 mmol/mol) or over, did not follow up with physicians within six months (Table 2).

\section{Frequency of follow-up with physicians treating pre-existing conditions}

For participants being treated for hypertension ( $n=640)$ or dyslipidaemia $(n=410), 367$ (57\%) of those with hypertension and 270 (66\%) of those with dyslipidaemia followed up with physicians after screening for diabetes. Most of these participants followed up with the physicians who were already treating them (80\%: hypertension, 90\%: dyslipidaemia).

\section{Predictors of no follow-up}


Table 3 shows age-adjusted analyses for predicting no follow-ups. Lower BMI, lower glucose level and negative proteinuria predicted no follow-ups in men, while treatment for comorbidities (hypertension, dyslipidaemia and depression) facilitated follow-ups. Lower glucose level, negative proteinuria and insured status predicted no follow-ups in women, while treatment for comorbidities (hypertension and dyslipidaemia) facilitated follow-ups.

Table 4 shows the adjusted ORs and 95\% CIs for no follow-ups by each variable. This multiple logistic regression analysis revealed that younger age, lower BMI, lower glucose level and negative proteinuria significantly predicted no follow-ups in men, while treatment for comorbidities (hypertension, dyslipidaemia and depression) facilitated follow-ups. For women, younger age and lower glucose level predicted no follow-ups, while treatment for comorbidities (dyslipidaemia) facilitated follow-ups. Areas under the ROC curve for the multiple logistic regression models were $0.72,0.73$ and 0.74 for men and $0.74,0.76$ and 0.77 for women at three, six and 12 months, respectively.

\section{Discussion}

We showed that 2,527 of 3,878 individuals (65.2\%; $65.9 \%$ of men and $59.4 \%$ of women) who were hyperglycaemic without treatment at the time of screening did not follow up with physicians within six months after the screening. Younger age, lower blood glucose level, lower BMI and negative proteinuria predicted no follow-ups, while treatment for comorbidities (hypertension, 
dyslipidaemia or depression) facilitated follow-ups. Our study is the first to reveal the frequency and predictors of no follow-ups in the Japanese diabetes screening system using a large database that links health claim data with mass screening results.

Direct evidence of the effectiveness of diabetes screening and its clinical implementation is lacking (Key Question 1 in Figure 1), although there have been several previous studies addressing this ${ }^{7-14,23,24}$. The U.S. Preventive Services Task Force summarizes screening for type 2 diabetes mellitus in adults as a grade I statement, meaning that current evidence is insufficient to assess the service ${ }^{7}$. A modelling study simulated that screening for type 2 diabetes would be cost-effective when initiated between the ages of 30 and 45 years, and repeated every three to five years ${ }^{25}$. Another study suggested that screening for type 2 diabetes and early intensive multifactorial treatment were feasible in general practice ${ }^{13}$. Adverse outcomes from diabetes screenings were not apparent in previous studies ${ }^{26-29}$, possibly because the studies did not sufficiently analyse dropout cases. Focus should also be placed on these cases in order to increase the effectiveness of diabetes screenings.

The screening attendance rate in the present study was $46 \%$ (Figure 2), and was $78 \%$ among insured participants and 29\% among dependents, which was similar to results of a Japanese national survey that showed 62\% of citizens attended a general health screening (employed: 69\%, unemployed: $49 \%)^{30}$. The screenings involved measurements of HbA1c and fasting or casual 
blood glucose to identify individuals who are possibly diabetic. Participants were instructed to fast beginning at the night before the screening, and for those who failed to maintain the fasting requirement, casual blood glucose was measured and the time since the last meal was recorded. In the present study, only HbA1c and fasting glucose were used to identify participants because these measurements are more stable than that of casual blood glucose. Participants were considered to be potentially diabetic if they had values above the cut-off for HbA1c and/or fasting glucose.

The rate of no follow-ups after screening was much higher in the present study (step 2 in Figure 1) than in national data showing that $39 \%$ of people with diabetes were not being treated ${ }^{6}$. This discrepancy can be attributed to the difference in research design. The national survey ${ }^{6}$ was based on self-report and could be affected by information bias, whereas our study used objective administrative data from health insurance claims and screenings. Moreover, the sampling process differed in that participants of the national survey were those who answered 'I have never been treated' or 'I was treated in the past, but not currently' to the question 'have you ever been referred to as a diabetic at a screening or at a medical institution?' In contrast, we asked the question "how many individuals did not follow up after screening positive for potential diabetes.” Another previous study reported a 50\% response rate to a pre-screening questionnaire for diabetes, and $77 \%$ of respondents at high risk for diabetes visited clinics ${ }^{15}$. Although the follow-up rate after screening was higher in the previous study than in ours, it was conducted in an interventional 
study setting and did not represent actual behaviour after screenings. Also, while it has been suggested that screening programs should follow-up on positive tests ${ }^{31}$, specific goals have not been set. It may be worth examining a concrete follow-up process to screening in the future. Even with moderate to severe hyperglycaemia, considerable proportions of participants did not follow up with physicians (Table 2). Surprisingly, one third of participants with an HbA1c value of 10\% (86 mmol/mol) or over failed to follow up with physicians. This highlights the urgent need for screeners to develop an effective reminder/recall system for such individuals. In addition, potential barriers to following up, e.g., pressure against leave work to visit doctors, need to be further investigated.

Our study identified a number of predictors for no follow-ups. The first was younger age. Younger people may lack awareness regarding the risks of hyperglycaemia and are less health-conscious than older people. Most of the younger people were working, and would not go to clinics if they were experiencing no symptoms or only mild symptoms because of their environment. This finding was consistent with that of the previous study ${ }^{15}$.

Second, lower BMI predicted no follow-ups in men. Some studies reported that obese people tend to visit general practice physicians more often than non-obese people ${ }^{32,33}$. Although our study was conducted in a screening setting, our results support the generalization that obese men are more likely to follow up with physicians than non-obese men. There are two possible reasons for 
this: (1) obesity increases the risk of diseases that require treatment, and (2) obesity increases a patient's sense of risk and leads to follow-up with physicians. On the other hand, obese women tended not to follow up with physicians. This implies that obese women might be less health conscious than obese men, and the tendency of obese individuals to engage in healthy behaviours, including follow-ups with physicians, might be influenced by gender. Examination of this hypothesis is beyond the scope of our study and further study will be needed to address this issue.

Third, lower blood glucose level predicted no follow-ups. This is related to a lack of risk awareness, similar to that found for age differences. Also, the above-mentioned study suggested that dropouts at the screening step were more likely to have lower risk scores on the pre-screening questionnaire $^{15}$.

Fourth, having or being treated for comorbidities including hypertension, dyslipidaemia, proteinuria and depression tended to facilitate follow-ups. Furthermore, over $80 \%$ of people who had been treated for hypertension or dyslipidaemia visited the same physicians after screening as hyperglycaemic. Treatment for comorbidities, particularly hypertension and dyslipidaemia, increased the likelihood that people who were hyperglycaemic at screening would follow up with physicians and receive clinical care. This suggests that many patients tend to attend the same clinics as part of the free access system in Japan. A previous study reported that patients with type 2 diabetes who are depressed poorly adhere to self-care ${ }^{16}$; however, the definition of depression in 
this report was based only on self-reported data, which is different from the definition adopted in our study. Our study participants who were classified as depressed were undergoing treatment and might have been more inclined to comply with instructions after screening. A lack of risk awareness, as discussed above, may be associated with proteinuria as well.

Sensitivity analyses was also conducted to examine differences in follow-up periods. When setting three or 12 months as the follow-up period, all explanatory variables showed similar trends as those for the six-month follow-up. Moreover, multiple logistic regression models and ROC curves were used to assess the ability of these models to predict outcomes. AUCs showed acceptable discrimination, with values ranging from 0.72 to 0.77 .

There are some limitations to this study. First, smoking and alcohol consumption were not included in the analysis due to missing data. Consequently, this study may not have considered potentially important information on behavioural factors. Second, as information on socioeconomic status was not available, we used the type of health insurance plan and insured/dependent status as surrogates for socioeconomic status. In Japan, the particular company one works for dictates the health insurance plan one has. While the type of health insurance plan and insured/dependent status have limitations as surrogates of socioeconomic status, the focus of the present study was the middle-aged working class of company employees, rather than a representative population comprising socially diverse sub-populations from around Japan. Given 
this design, our study population could be considered more socially homogeneous than a national representative population. The existence of unadjusted variables (i.e., residual confounding) may have limited the fitness of the obtained models. Third, the analysed data were only from three employee-based health insurance plans. As a result, the participants were younger than the general Japanese population and the number of women was limited. Therefore, extrapolation of the present findings to other Japanese populations, especially elderly people requires careful consideration. On the other hand, recent clinical guidelines ${ }^{34}$ have reconsidered the strict glycaemic goals for treating older adults with diabetes, and aggressive screening for older adults to detect possible diabetes remains controversial ${ }^{35,}{ }^{36}$. Against this backdrop, our focus on a younger population (working-age adults) would contribute to maintaining work productivity in society.

In conclusion, we found that two-thirds of participants who were hyperglycaemic did not follow up with physicians after screening. Younger age, lower BMI, lower blood glucose level and negative proteinuria predicted no follow-ups, and treatment for comorbidities including hypertension, dyslipidaemia and depression facilitated follow-ups. To improve the screening system and health care cost efficiency, a more effective follow-up process that includes recommendations to visit physicians must be offered to those at high risk of not following up. Further studies will be needed to accurately evaluate diabetes screening, including no attendance or no follow-ups, which will contribute to a more effective health care system. 


\section{Conflict of interest}

The authors declare that they have no conflict of interest.

\section{Acknowledgments}

This work was partly supported by a grant-in aid from the Ministry of Education, Culture, Sports,

Science and Technology, Japan [Challenging Exploratory Research 24659320]. 


\section{References}

[1] Stratton IM, Adler AI, Neil HA, Matthews DR, Manley SE, Cull CA, et al. Association of glycaemia with macrovascular and microvascular complications of type 2 diabetes (UKPDS 35): prospective observational study. BMJ. 2000; 321(7258): 405-12.

[2] Rubin RR, Peyrot M. Quality of life and diabetes. Diabetes/metabolism research and reviews. 1999; 15(3): 205-18.

[3] American Diabetes Association. Economic costs of diabetes in the U.S. in 2012. Diabetes Care. 2013; 36(4): 1033-46.

[4] Guariguata L, Whiting DR, Hambleton I, Beagley J, Linnenkamp U, Shaw JE. Global estimates of diabetes prevalence for 2013 and projections for 2035. Diabetes Res Clin Pract. 2014; 103(2): 137-49.

[5] Roglic G, Unwin N. Mortality attributable to diabetes: estimates for the year 2010. Diabetes Res Clin Pract. 2010; 87(1): 15-9.

[6] Health, Labour and Welfare Statistics Association. Kokumin eisei no doukou kousei no sihyou 2012/2013. Journal of Health and Welfare Statistics.2012; 59(9).

[7] U.S. Preventive Services Task Force. Screening for Type 2 Diabetes Mellitus in Adults. 2008. Available from: http://www.uspreventiveservicestaskforce.org/uspstf/uspsdiab.htm. (accessed on 30 March 2014)

[8] Norris SL, Kansagara D, Bougatsos C, Nygren P, Fu R. Screening for Type 2 Diabetes Mellitus: Update of 2003 Systematic Evidence Review for the U.S. Preventive Services Task 
Force.

[9] Wareham NJ, Griffin SJ. Should we screen for type 2 diabetes? Evaluation against National Screening Committee criteria. BMJ. 2001; 322(7292): 986-8.

[10] Engelgau MM, Narayan KM, Herman WH. Screening for type 2 diabetes. Diabetes Care. 2000; 23(10): 1563-80.

[11] Hoerger TJ, Harris R, Hicks KA, Donahue K, Sorensen S, Engelgau M. Screening for type 2 diabetes mellitus: a cost-effectiveness analysis. Ann Intern Med. 2004; 140(9): 689-99.

[12] The cost-effectiveness of screening for type 2 diabetes. CDC Diabetes Cost-Effectiveness Study Group, Centers for Disease Control and Prevention. JAMA. 1998; 280(20): 1757-63.

[13] Griffin SJ, Borch-Johnsen K, Davies MJ, Khunti K, Rutten GE, Sandbaek A, et al. Effect of early intensive multifactorial therapy on 5-year cardiovascular outcomes in individuals with type 2 diabetes detected by screening (ADDITION-Europe): a cluster-randomised trial. Lancet. 2011; 378(9786): 156-67.

[14] Schellhase KG, Koepsell TD, Weiss NS, Wagner EH, Reiber GE. Glucose screening and the risk of complications in Type 2 diabetes mellitus. Journal of clinical epidemiology. 2003; 56(1): 75-80.

[15] Christensen JO, Sandbaek A, Lauritzen T, Borch-Johnsen K. Population-based stepwise screening for unrecognised Type 2 diabetes is ineffective in general practice despite reliable algorithms. Diabetologia. 2004; 47(9): 1566-73.

[16] Gonzalez JS, Safren SA, Cagliero E, Wexler DJ, Delahanty L, Wittenberg E, et al. 
Depression, self-care, and medication adherence in type 2 diabetes: relationships across the full range of symptom severity. Diabetes Care. 2007; 30(9): 2222-7.

[17] Ikegami N, Yoo BK, Hashimoto H, Matsumoto M, Ogata H, Babazono A, et al. Japanese universal health coverage: evolution, achievements, and challenges. Lancet. 2011; 378(9796): 1106-15.

[18] Ikeda N, Saito E, Kondo N, Inoue M, Ikeda S, Satoh T, et al. What has made the population of Japan healthy? Lancet 2011; 378(9796): 1094-105.

[19] Ministry of Health, Labour and Welfare. Standard Program about the medical screening and the health instruction. Available from: http://www.mhlw.go.jp/bunya/kenkou/seikatsu/pdf/02a.pdf (accessed on 30 March 2014)

[20] Kimura S, Sato T, Ikeda S, Noda M, Nakayama T. Development of a database of health insurance claims: standardization of disease classifications and anonymous record linkage. $\mathrm{J}$ Epidemiol. 2010; 20(5): 413-9.

[21] Atsunori Kashiwagi MK, Eiichi Araki, Yoshitomo Oka, Toshiaki Hanafusa, Hiroshi, Ito MT, Shinichi Oikawa, et al. International clinical harmonization of glycated haemoglobin in Japan : From Japan Diabetes Society to National Glycohaemoglobin Standardization Program values. Diabetology International. 2012; 3(1): 39-40.

[22] WHO Collaborating Centre for Drug Statistics Methodology. ATC/DDD Index 2014. Available from: http://www.whocc.no/atc_ddd_index/ (accessed on 30 March 2014)

[23] Agarwal S, Raman R, Kumari RP, Deshmukh H, Paul PG, Gnanamoorthy P, et al. Diabetic 
retinopathy in type II diabetics detected by targeted screening versus newly diagnosed in general practice. Ann Acad Med Singapore. 2006; 35(8): 531-5.

[24] Glumer C, Yuyun M, Griffin S, Farewell D, Spiegelhalter D, Kinmonth AL, et al. What determines the cost-effectiveness of diabetes screening? Diabetologia. 2006; 49(7):1536-44.

[25] Kahn R, Alperin P, Eddy D, Borch-Johnsen K, Buse J, Feigelman J, et al. Age at initiation and frequency of screening to detect type 2 diabetes: a cost-effectiveness analysis. Lancet. 2010; 375(9723): 1365-74.

[26] Paddison CA, Eborall HC, French DP, Kinmonth AL, Prevost AT, Griffin SJ, et al. Predictors of anxiety and depression among people attending diabetes screening: a prospective cohort study embedded in the ADDITION (Cambridge) randomized control trial. British journal of health psychology. 2011; 16(Pt 1): 213-26.

[27] Paddison CA, Eborall HC, Sutton S, French DP, Vasconcelos J, Prevost AT, et al. Are people with negative diabetes screening tests falsely reassured? Parallel group cohort study embedded in the ADDITION (Cambridge) randomised controlled trial. BMJ. 2009; 339: b4535.

[28] Eborall HC, Griffin SJ, Prevost AT, Kinmonth AL, French DP, Sutton S. Psychological impact of screening for type 2 diabetes: controlled trial and comparative study embedded in the ADDITION (Cambridge) randomised controlled trial. BMJ. 2007; 335(7618): 486.

[29] Park P, Simmons RK, Prevost AT, Griffin SJ. Screening for type 2 diabetes is feasible, acceptable, but associated with increased short-term anxiety: a randomised controlled trial in British general practice. BMC Public Health. 2008; 8: 350. 
[30] Health, Labour and Welfare Statistics Association. Kokumin eisei no doukou kousei no sihyou 2010/2011. Journal of Health and Welfare Statistics 2010; 57(9).

[31] Spasoff RA. Epidemiologic Methods for Health Policy: Oxford University Press, Inc.; 1999.

[32] van Dijk L, Otters HB, Schuit AJ. Moderately overweight and obese patients in general practice: a population based survey. BMC Fam Pract. 2006; 7: 43.

[33] Wildenschild C, Kjoller M, Sabroe S, Erlandsen M, Heitmann BL. Change in the prevalence of obesity and use of health care in Denmark: an observational study. Clinical epidemiology. 2011; 3: 31-41.

[34] American Diabetes Association. Standards of medical care in diabetes-2012. Diabetes Care. 2012; 35 suppl 1:S11-63.

[35] Hoerger TJ, Harris R, Hicks KA, Donahue K, Sorensen S, Engelgau M. Screening for type 2 diabetes mellitus: a cost-effectiveness analysis. Ann Intern Med. 2004;140:689-99.

[36] Waugh N, Scotland G, McNamee P, Gillett M, Brennan A, Goyder E, et al. Screening for type 2 diabetes: literature review and economic modelling. Health Technol Assess. 2007;11:iii-iv, ix-xi, 1-125. 


\section{Figure legends}

Fig. 1 - Japanese diabetes screening process.

Note: Screenings are primarily taken place at work sites or local community facilities, and

screened patients are advised to follow up with physicians after screening.

KQ, key question(s);

KQ1: Is there direct evidence that systematic screening for diabetes among asymptomatic adults

improves health outcomes?

KQ2: Does follow-up with physicians early after screening for diabetes provide an incremental

benefit in health outcomes?

KQ3: Does initiating early treatment for type 2 diabetes as a result of screening provide an

incremental benefit in health outcomes ?

Does initiating early treatment of impaired fasting glucose or impaired glucose tolerance as a

result of screening provide an incremental benefit in final health outcomes?

Step 1: Attending diabetes screening

Step 2: Follow-up with physicians after screening

Step 3: Initiation of appropriate treatment 
Fig.2 - Flowchart of the study participants.

$\mathrm{HbA}_{1 c}$, glycated haemoglobin 


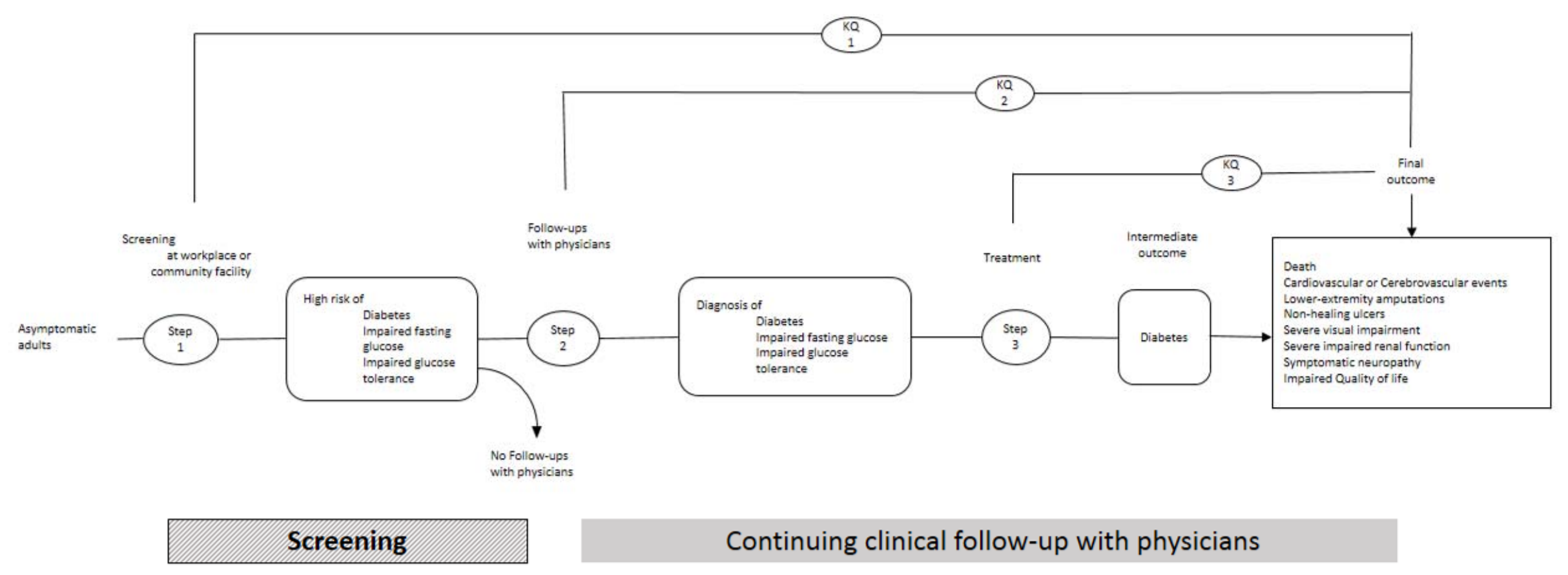

Fig. 1 


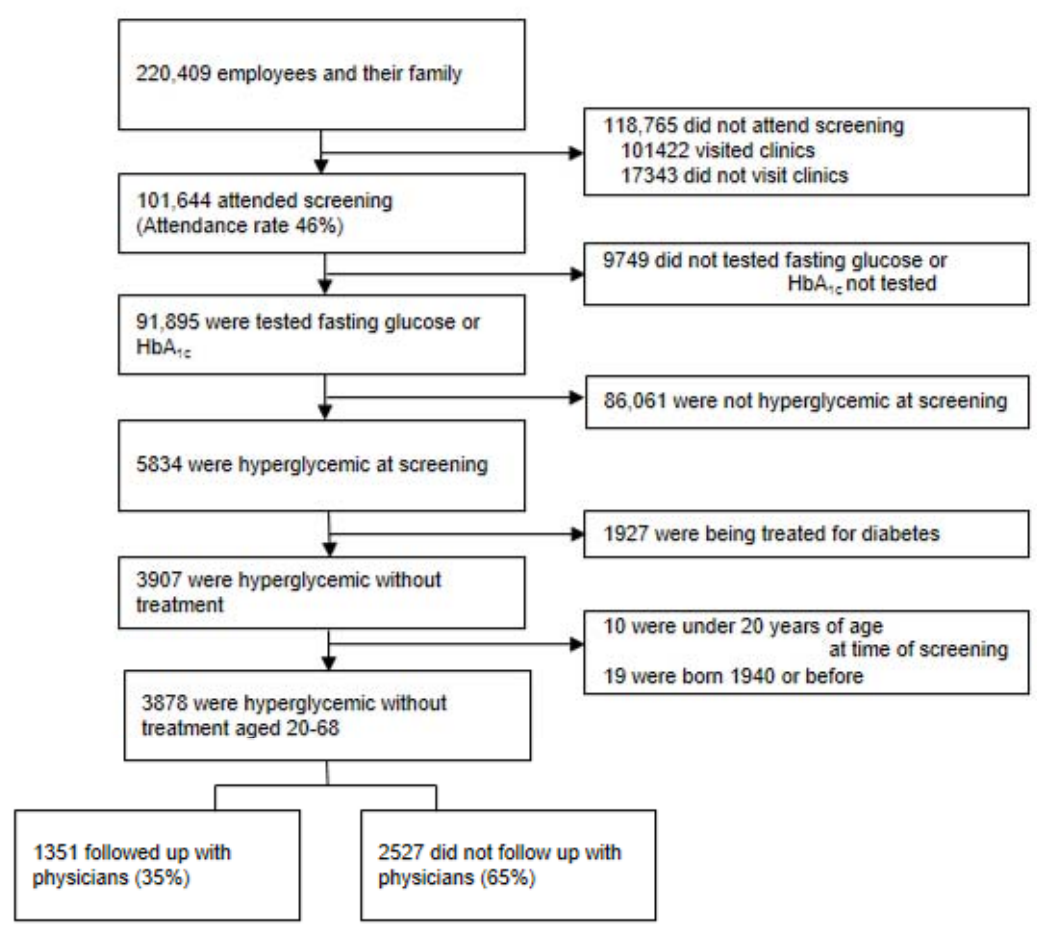

Fig. 2 
Table 1 - Characteristics of 3878 participants with hyperglycemia, stratified by gender

\begin{tabular}{|c|c|c|}
\hline & Men (n=3420) & Women $(n=458)$ \\
\hline \multicolumn{3}{|l|}{ No follow-ups with physicians, n (\%) } \\
\hline $0-3$ months & $2563(74.9)$ & 307 (67.0) \\
\hline 0-6 months & 2255 (65.9) & $272(59.4)$ \\
\hline 0-12 months & 1989 (58.2) & 246 (53.7) \\
\hline Mean age (SD), years & $47(10)$ & $47(11)$ \\
\hline \multicolumn{3}{|l|}{ Age groups, n (\%) } \\
\hline 20-24 years & $43(1.3)$ & $7(1.5)$ \\
\hline 25-29 years & $53(1.6)$ & $12(2.6)$ \\
\hline 30-34 years & $334(9.8)$ & $45(9.8)$ \\
\hline 35-39 years & $429(12.5)$ & $63(13.8)$ \\
\hline $40-44$ years & $542(15.9)$ & $73(15.9)$ \\
\hline $45-49$ years & $539(15.8)$ & $41(9.0)$ \\
\hline 50-54 years & $433(12.7)$ & $65(14.2)$ \\
\hline $55-59$ years & $607(17.8)$ & $83(18.1)$ \\
\hline 60-64 years & $414(12.1)$ & $60(13.1)$ \\
\hline 65-68 years & $26(0.76)$ & $9(2.0)$ \\
\hline Mean body mass index (SD), $\mathrm{kg} / \mathrm{m}^{2} \mathrm{a}$ & $25.1(4.4)$ & $25.0(5.4)$ \\
\hline
\end{tabular}


Mean fasting glucose (SD), $\mathrm{mg} / \mathrm{dl}^{\mathrm{b}}$

Mean $\mathrm{HbA}_{1 \mathrm{c}}$ (SD), \%c

Glycosuria, n (\%)

Proteinuria, n (\%) ${ }^{d}$

Hypertension, n (\%)

Dyslipidemia, n (\%)

Depression, n (\%)

Smoking, n (\%)

Alcohol consumption, n (\%)

Everyday

Sometimes

Rarely

Missing data

Mean haemoglobin (SD), g/dlg

Health insurance plans, n (\%)

A

B

C

Insured status at the health insurance plans, n (\%)
$141.0(34.5)$

$135.7(38.0)$

$$
6.5(1.3)
$$

$6.6(1.4)$

$758(22.2)$

63 (13.8)

223 (6.5)

$26(5.7)$

577 (16.9)

63 (13.8)

357 (10.4)

53 (11.6)

$8(0.2)$

$3(0.7)$

1383 (40.4)

35 (7.6)

493 (14.4)

$9(2.0)$

772 (22.6)

38 (8.3)

897 (26.2)

$262(57.2)$

1258 (36.8)

149 (32.5)

$15.2(1.2)$

$13.3(1.6)$

612 (17.9)

104 (22.7)

2702 (79.0)

343 (74.9)

106 (3.1)

$11(2.4)$ 
aata missing for 1 man.

bData missing for 497 men and 18 women.

${ }^{\mathrm{c} D a t a}$ missing for 89 men and 6 women.

${ }^{\mathrm{d}}$ Data missing for 149 men and 7 women.

${ }^{\mathrm{e}}$ Data missing for 787 men and 113 women.

${ }^{\mathrm{f}}$ Data missing for 1258 men and 149 women.

'Data missing for 109 men and 49 women. 
Table 2 - No follow-ups with physicians after screening, stratified by HbA1c

\begin{tabular}{|c|c|c|c|c|c|c|c|c|c|}
\hline \multirow{3}{*}{ HbA1c } & \multicolumn{9}{|c|}{ No follow-ups with physicians } \\
\hline & & 3 months & & & 6 months & & & 12 months & \\
\hline & total, $N(\%)$ & men, $N(\%)$ & women, $N(\%)$ & total, $N(\%)$ & men, $N(\%)$ & women, $N(\%)$ & total, $N(\%)$ & men, $N(\%)$ & women, $N(\%)$ \\
\hline$<6 \%$ & 1471(84) & 1332(85) & 139(78) & $1394(80)$ & $1255(80)$ & 139 (78) & $1296(74)$ & 1165(74) & 131(73) \\
\hline $6 \% \leq,<7 \%$ & 1063(69) & $935(70)$ & $128(62)$ & 875 (57) & $771(58)$ & $104(50)$ & 735(47) & $646(48)$ & 89(43) \\
\hline $7 \% \leq,<8 \%$ & $165(57)$ & $146(58)$ & $19(53)$ & $126(44)$ & $110(43)$ & $16(44)$ & $97(34)$ & 83(33) & $14(39)$ \\
\hline $8 \% \leq,<10 \%$ & $120(61)$ & 111(62) & $9(56)$ & $96(49)$ & $89(49)$ & $7(44)$ & $80(41)$ & $74(41)$ & $6(38)$ \\
\hline $10 \% \leq$ & $46(46)$ & $39(51)$ & $7(37)$ & $35(35)$ & $29(36)$ & $6(32)$ & $27(27)$ & $21(26)$ & $6(32)$ \\
\hline
\end{tabular}


Table 3 - Age-adjusted Odds Ratios for No Follow-ups, Stratified by Gender

\begin{tabular}{|c|c|c|c|c|c|c|}
\hline & \multicolumn{3}{|c|}{ Men } & \multicolumn{3}{|c|}{ Women } \\
\hline & 3 months & 6 months & 12months & 3 months & 6 months & 12months \\
\hline & OR $(95 \% \mathrm{CI})^{\mathrm{a}}$ & OR $(95 \% \mathrm{CI})^{\mathrm{a}}$ & OR $(95 \% \mathrm{CI})^{\mathrm{a}}$ & OR $(95 \% \mathrm{CI})^{\mathrm{a}}$ & OR $\left(95 \%\right.$ CI) ${ }^{\mathrm{a}}$ & OR $(95 \% \mathrm{CI})^{\mathrm{a}}$ \\
\hline Body mass index, $1 \mathrm{~kg} / \mathrm{m} 2$ increase & $0.95(0.93-0.96)$ & $0.93(0.91-0.94)$ & $0.92(0.91-0.94)$ & $0.98(0.94-1.02)$ & $0.97(0.93-1.00)$ & $0.96(0.92-0.99)$ \\
\hline Fasting glucose, $10 \mathrm{mg} / \mathrm{dl}$ increase & 0.93(0.91-0.95) & $0.95(0.93-0.97)$ & $0.95(0.93-0.97)$ & $0.97(0.92-1.02)$ & $0.97(0.92-1.02)$ & $0.99(0.94-1.04)$ \\
\hline $\mathrm{HbA}_{1 \mathrm{c}}, 1 \%$ increase & $0.69(0.65-0.73)$ & $0.65(0.61-0.69)$ & $0.61(0.57-0.65)$ & $0.74(0.64-0.87)$ & $0.68(0.58-0.81)$ & $0.70(0.59-0.84)$ \\
\hline Glycosuria & $0.59(0.49-0.70)$ & $0.59(0.50-0.69)$ & $0.55(0.46-0.64)$ & $0.29(0.17-0.52)$ & $0.25(0.14-0.46)$ & $0.28(0.15-0.51)$ \\
\hline Proteinuria & $0.54(0.41-0.72)$ & $0.50(0.38-0.66)$ & $0.46(0.35-0.61)$ & $0.32(0.14-0.73)$ & $0.39(0.17-0.92)$ & $0.42(0.18-1.02)$ \\
\hline Hypertension & $0.43(0.35-0.52)$ & $0.39(0.32-0.47)$ & $0.35(0.29-0.43)$ & $0.55(0.31-0.96)$ & $0.44(0.24-0.79)$ & 0.19(0.09-0.39) \\
\hline Dyslipidemia & $0.28(0.22-0.35)$ & $0.28(0.22-0.35)$ & $0.25(0.19-0.32)$ & $0.39(0.21-0.72)$ & $0.28(0.14-0.56)$ & $0.23(0.93-0.97)$ \\
\hline Depression & $0.50(0.37-0.67)$ & $0.18(0.04-0.90)$ & $0.43(0.32-0.58)$ & $0.81(0.38-1.71)$ & $0.29(0.02-3.47)$ & $0.58(0.27-1.24)$ \\
\hline Smoking & $1.02(0.87-1.20)$ & $0.93(0.80-1.07)$ & $0.93(0.81-1.07)$ & $0.79(0.37-1.68)$ & $1.11(0.52-2.37)$ & $1.09(0.52-2.28)$ \\
\hline \multicolumn{7}{|l|}{ Alcohol consumption } \\
\hline Everyday & 1.00 (Reference) & 1.00 (Reference) & 1.00 (Reference) & 1.00 (Reference) & 1.00 (Reference) & 1.00 (Reference) \\
\hline Sometimes & $0.80(0.62-1.03)$ & $0.87(0.69-1.10)$ & $0.79(0.63-0.10)$ & $2.60(0.58-11.58)$ & $2.91(0.62-13.7)$ & 2.06(0.44-9.66) \\
\hline Rarely & $0.86(0.67-1.12)$ & $0.91(0.72-1.14)$ & $0.83(0.67-1.05)$ & $2.01(0.52-7.73)$ & $2.25(0.55-9.29)$ & $1.84(0.45-7.63)$ \\
\hline Haemoglobin (g/dl) & $1.02(0.95-1.08)$ & $0.98(0.92-1.04)$ & $0.96(0.90-1.01)$ & $0.98(0.86-1.11)$ & $0.93(0.82-1.06)$ & $0.96(0.85-1.09)$ \\
\hline \multicolumn{7}{|l|}{ Health insurance plan } \\
\hline A & 1.00 (Reference) & 1.00 (Reference) & 1.00 (Reference) & 1.00 (Reference) & 1.00 (Reference) & 1.00 (Reference) \\
\hline B & $1.12(0.91-1.36)$ & $1.21(1.01-1.46)$ & $1.25(1.04-1.50)$ & $0.80(0.49-1.31)$ & $0.94(0.59-1.50)$ & 1.15(0.73-1.84) \\
\hline C & $0.54(0.35-0.84)$ & $0.82(0.54-1.25)$ & $0.74(0.48-1.12)$ & $1.04(0.26-4.25)$ & $0.71(0.20-2.55)$ & $1.08(0.30-3.87)$ \\
\hline Dependent status at health insurance plans & NA & NA & NA & $0.57(0.36-0.90)$ & $0.60(0.39-0.92)$ & $0.49(0.32-0.75)$ \\
\hline
\end{tabular}

NA, Not applicable. 


\section{${ }^{a}$ Adjusted for age.}


Table 4 - Adjusted Odds Ratios for No Follow-ups, Stratified by Gender

\begin{tabular}{|c|c|c|c|c|c|c|}
\hline & \multicolumn{3}{|c|}{ Men } & \multicolumn{3}{|c|}{ Women } \\
\hline & 3 months & 6 months & 12months & 3 months & 6months & 12 months \\
\hline & OR $(95 \% \text { CI })^{\mathrm{a}}$ & OR $(95 \% \text { CI })^{\mathrm{a}}$ & OR $(95 \% \text { CI })^{\mathrm{a}}$ & OR $(95 \% \mathrm{CI})^{\mathrm{b}}$ & OR $(95 \% \mathrm{CI})^{\mathrm{b}}$ & OR $(95 \% \mathrm{CI})^{\mathrm{b}}$ \\
\hline Age, 10 year increase & $0.82(0.75-0.90)$ & $0.78(0.71-0.85)$ & $0.76(0.70-0.83)$ & $0.82(0.64-1.03)$ & $0.72(0.57-0.91)$ & $0.76(0.61-0.95)$ \\
\hline Body mass index, 1 kg/m2 increase & $0.999(0.98-1.02)$ & $0.979(0.960-0.998)$ & $0.98(0.96-0.99)$ & $1.02(0.97-1.06)$ & $1.006(0.965-1.049)$ & $1.001(0.96-1.043)$ \\
\hline $\mathrm{HbA}_{1 \mathrm{c}}, 1 \%$ increase & $0.69(0.65-0.74)$ & $0.67(0.62-0.71)$ & $0.64(0.59-0.69)$ & $0.78(0.65-0.92)$ & $0.71(0.59-0.85)$ & 0.74(0.61-0.89) \\
\hline Proteinuria & $0.73(0.54-0.99)$ & $0.72(0.54-0.97)$ & $0.69(0.51-0.94)$ & $0.369(0.15-0.91)$ & $0.53(0.21-1.34)$ & $0.62(0.24-1.64)$ \\
\hline Hypertension & $0.55(0.44-0.68)$ & $0.49(0.40-0.61)$ & $0.45(0.37-0.56)$ & $0.67(0.36-1.28)$ & $0.57(0.30-1.09)$ & $0.24(0.11-0.52)$ \\
\hline Dyslipidemia & $0.36(0.28-0.46)$ & $0.38(0.30-0.49)$ & $0.35(0.27-0.46)$ & $0.39(0.20-0.76)$ & $0.31(0.15-0.63)$ & $0.30(0.13-0.69)$ \\
\hline Depression & $0.17(0.04-0.78)$ & $0.14(0.03-0.74)$ & $0.20(0.04-1.07)$ & $0.19(0.016-2.31)$ & $0.37(0.03-4.85)$ & $0.41(0.03-5.22)$ \\
\hline \multicolumn{7}{|l|}{ Health insurance plan } \\
\hline A & 1.00 (Reference) & 1.00 (Reference) & 1.00 (Reference) & 1.00 (Reference) & 1.00 (Reference) & 1.00 (Reference) \\
\hline B & 0.85(0.69-1.06) & 0.89 (0.73- 1.09) & 0.89(0.73-1.09) & $0.58(0.33-1.001)$ & $0.69(0.40-1.18)$ & $0.76(0.44-1.31)$ \\
\hline $\mathrm{C}$ & $0.50(0.32-0.78)$ & $0.77(0.49-1.20)$ & $0.69(0.44-1.07)$ & $0.71(0.15-3.34)$ & $0.54(0.13-2.21)$ & $0.69(0.17-2.87)$ \\
\hline Dependent status at health insurance plans & NA & NA & NA & $0.63(0.36-1.11)$ & $0.74(0.43-1.27)$ & $0.61(0.35-1.05)$ \\
\hline
\end{tabular}

NA, Not applicable.

${ }^{a}$ Adjusted for age, proteinuria, $\mathrm{HbA}_{1 \mathrm{c}}$, BMI, hypertension, dyslipidemia, depression, health insurance plan.

${ }^{\mathrm{b}}$ Adjusted for age, proteinuria, $\mathrm{HbA}_{1 \mathrm{c}}$, BMI, hypertension, dyslipidemia, depression, health insurance plan, and dependent status at the health insurance plan. 